\title{
Gender Differences in Chronic HBsAg Carriers in Italy: Evidence for the Independent Role of Male Sex in Severity of Liver Disease
}

\author{
Tommaso Stroffolini, ${ }^{1}$ Rozenn Esvan, ${ }^{1}$ Elisa Biliotti, ${ }^{1 *}$ Evangelista Sagnelli, ${ }^{2}$ \\ Giovanni Battista Gaeta, ${ }^{3}$ and Piero Luigi Almasio ${ }^{4}$ \\ ${ }^{1}$ Department of Tropical and Infectious Diseases, University of Rome, Italy \\ ${ }^{2}$ Department of Public Medicine, Infectious Diseases Unit, Second University of Naples, Caserta CE, Italy \\ ${ }^{3}$ Department of Internal and Specialized Medicine, Second University of Naples, Caserta CE, Italy \\ ${ }^{4}$ Department of Gastroenterology, University of Palermo, Italy
}

It has been shown that sexual hormones have an opposite effect on hepatic fibrosis progression and hepatocellular carcinoma development. Sex differences among 2,762 chronic HBsAg carriers consecutively referring Italian hospitals in 2001 and in 2007 have been evaluated, particularly focusing on the role of gender on severity of liver disease. The overall sex ratio (males/females) was 2.6. Females were more likely born abroad and new diagnosis cases; but less likely HIV coinfected. No sex difference was observed regarding coinfection with other hepatitis viruses. The sex ratio linearly increased with increasing severity of liver disease, being 1.3 in normal ALT, 2.8 in chronic hepatitis, 3.6 in liver cirrhosis, and 6.8 in hepatocellular carcinoma. Adjustment by multiple logistic regression analysis for the confounding effect of age, alcohol intake, HDV infection, HCV infection, and BMI shows that male gender is an independent predictor of the likelihood of more severe liver disease (O.R. 1.7; C.I. $95 \%=1.3-2.1$ ). HBV-DNA levels resulted not associated with the outcome of chronic HBV infection. Despite some potential risk factors associated with liver disease, such as HBV genotype or mutations, not having been controlled for due to lack of availability, the observed sex disparity in the outcome of chronic HBV infection may support biological obervation that HBV infection could be considered a sex hormone-responsive virus. J. Med. Virol. 87:1899-1903, 2015.

(C) 2015 Wiley Periodicals, Inc.

KEY WORDS: Chronic HBsAg carriers; sex differences; cirrhosis; hepatocellular carcinoma

\section{INTRODUCTION}

Since several years, it is well known that men are more likely than women to be chronic carriers of Hepatitis B virus (HBV) [Blumberg et al., 1972]. This difference may be due either to the more frequent exposure to HBV in males, either to more likely HBV clearance, once infected, in females, either to both reasons.

Chronic hepatitis B virus infection appears to progress more rapidly in males than in females [McMahon et al., 1990; Chen 1993; Poynard et al., 2001]. The relative risk of chronic HBV-related endstage liver disease, hepatocellular carcinoma (HCC), and liver disease-related death are consistently several fold (range from 1.5 to 7.6 times) higher in males than in females [Lee et al., 1999; Evans et al., 2002; Chang et al., 2000; Taylor et al., 2009].

In the last few years, we have conducted two surveys on the characteristics of chronic hepatitis B surface antigen (HBsAg) carriers referring Italian hospitals [Sagnelli et al., 2008; Stroffolini et al., 2009]. It allows us a good opportunity to evaluate sex differences in chronic HBsAg carriers. Particular attention was addressed to evaluate the potential independent role, if any, of male sex in liver disease severity.

\section{PATIENTS AND METHODS}

The two studies have been previously described [Sagnelli et al., 2008; Stroffolini et al., 2009]. Briefly, the first study enrolled 1,366 chronic HBsAg carriers

${ }^{*}$ Correspondence to: Elisa Biliotti, M.D., Department of Tropical and Infectious Diseases, Sapienza University of Rome, Viale Del Policlinico 155, 00161 Rome.

E-mail: elisabiliotti@yahoo.it

Accepted 15 April 2015

DOI $10.1002 /$ jmv. 24243

Published online 2 June 2015 in Wiley Online Library

(wileyonlinelibrary.com). 
consecutively referring to 79 Italian hospitals (both tertiary and peripheral centers) from 1 February through July 31, 2001 [Sagnelli et al., 2008]. Hospitals were randomly selected from all Italian hospitals by a systematic cluster-sampling procedure. The second study, performed in 21 tertiary hospitals, recruited 1,396 HBsAg positive subjects consecutively observed from September 2006 to February 2007 [Stroffolini et al., 2009]. In both studies, subjects as either in-patients or out-patients represented the target population. "Incident cases" were defined those who were first diagnosed during the study period and had a negative anti-HBc IgM test. "Prevalent cases" were defined those observed during the study period but known to be HBsAg positive. For each subject, the demographic, clinical, and etiological data were recorded using a pre-coded questionnaire.

Hepatitis B serum markers (HBsAg, HBeAg, anti$\mathrm{HBe}$ ) and anti-HDV antibodies were determined by commercial immunoenzyme assays (Abbott Laboratories, North Chicago, IL). Antibodies to hepatitis C virus (anti-HCV) were detected by third-generation commercial immunoenzymatic assays (Ortho Diagnostic Systems, Raritan, NJ). Serum HBV DNA levels were assessed by polymerase chain reaction (PCR) amplification using different commercial assays available in 2007 (Cobas Ampliscreen HBV test, Roche; Procleix Ultrio assay, Chiron), with low detection limits of 600 copies/ml. HBV-DNA levels were only measured for the 2007 cohort.

Chronic hepatitis was diagnosed by liver histology or, for patients without liver biopsy, based on the presence of persistently ( $>6$ months) abnormal alanine aminotransferase (ALT) values in the absence of clinical, biochemical, and ultrasound markers of liver cirrhosis [Gaiani et al., 1997]. Cirrhosis was diagnosed by either liver biopsy or the presence of unequivocal clinical, biochemical, and ultrasound signs. Hepatocellular carcinoma (HCC) diagnosis was made by histology, imaging techniques or by $\alpha_{1}$-feto protein value greater than $400 \mathrm{ng} / \mathrm{ml}$ [Bruix and Sherman, 2005].

\section{Statistical Analysis}

Continuous variables were summarized as means and standard deviation, and categorical variables were summarized as absolute and relative frequencies. Differences in the means were evaluated on unpaired Student's $t$-test, and the Chi-squared test was applied to categorical variables. A $P$ value $<0.05$ was considered to be significant.

The crude odds ratios (O.R.), which are estimates of the relative risk linking severity of liver disease (i.e., liver cirrhosis and/or HCC) to potential risk factors, were calculated by univariate analysis. Adjusted O.R. were calculated by stepway multiple logistic regression analysis to identify variables that were independently associated with severity of liver disease. Only variables that resulted associated at univariate analysis were entered into the logistic model. In the logistic model, more severe liver disease was the outcome variable; age, sex, anti-delta positivity, anti-HCV positivity, alcohol intake, and body mass index resulted the independent variables. Adjustment for other potential confounding variables such as diabetes, HBV genotype, or presence of HBV genomic mutations was not performed, as these data were not available.

\section{RESULTS}

Overall 2,762 chronic HBsAg carriers have been evaluated. Comparison of the main characteristics of subjects enrolled in the two studies is shown in Table I. Mean age and sex distribution were quite similar. Incident cases were more likely represented in 2001, while people born abroad were more likely recruited in 2007. The proportion of subjects $\mathrm{HBeAg}$ positive and that of people coinfected with HDV or $\mathrm{HIV}$, was stable over time. The proportion of $\mathrm{HBsAg}$ positive cases tested for anti-HDV was very high, being $100 \%(1,366 / 1,366)$ in 2001 and $84.4 \%(1,179 /$ $1,396)$ in 2007. The proportion of people HCV coinfected strongly decreased over time from $16.3 \%$ in 2001 to $6.5 \%$ in $2007(P<0.01)$.

Table II reports the comparison by gender of the combined two studies according to different variables. The overall sex ratio was $2.6 \quad(2,000$ males/762 females). Females were more likely than males incident cases $(16.7 \%$ vs. $10.9 \%, P<0.01)$ and born abroad $(7.9 \%$ vs. $5.1 \%, P<0.04)$; but less likely HIV coinfected $(0.3 \%$ vs. $2.9 \%, P<0.01)$. A significant greater proportion $(P<0.01)$ of males had a more severe liver disease (i.e., liver cirrhosis and/or HCC). The sex ratio (M/F ratio) linearly increased according to the severity of liver disease, being 1.3 in subjects with normal ALT, 2.8 in those with chronic hepatitis, 3.6 in liver cirrhosis cases and 6.8 in those with HCC.

Table III reports the crude and the adjusted O.R. derived by multiple logistic regression analysis. At univariate analysis (crude O.R.) all the variables considered resulted associated with more severe liver diseases, but not highest HBV-DNA levels (O.R. 0.74; C.I. $95 \%=0.56-1.00$ ). After adjustment by multiple logistic regression analysis for the disturbing influence of the other variables considered, male sex (O.R. 1.67; C.I. 95\% $=1.34-2.06$ ) resulted an independent predictor of the likelihood of liver cirrhosis and/or HCC (other than age, HCV and HDV infection, and alcohol intake). Body mass index was not associated.

Comparison of male HBsAg positive carriers 6 years apart shows that the mean age of subjects (48.7 years vs. 49.9 years; $P<0.04$ ), the proportion of people born abroad (3.5\% vs. $6.5 \% ; P<0.05)$, and the proportion of liver cirrhosis cases $(19.7 \%$ vs. $22.27 \% ; P<0.05)$ significantly increased over time; while the proportion of incident cases $(13.8 \%$ vs. $8.4 \% ; P<0.01)$, and that of people $\mathrm{HCV}$ coinfected $(17.2 \%$ vs. $7.2 \%$; 
TABLE I. Comparison of Chronic HBsAg Carriers in Italy in Two Different Studies (2001 vs 2007)

\begin{tabular}{llrr}
\hline Factor & 2001 study $(\mathrm{n}=1,366)$ & 2007 study $(\mathrm{n}=1,396)$ & $P$ value \\
\hline Age (years) & $49.1 \pm 13.9$ & $49.7 \pm 12.8$ & N.S. \\
Gender male & $975(71.4 \%)$ & $1026(73.5 \%)$ & N.S. \\
Incident cases & $200(15.8 \%)$ & $132(9.5 \%)$ & $<0.01$ \\
Born abroad & $57(4.2 \%)$ & $104(7.4 \%)$ & $<0.01$ \\
HBeAg+ & $164(12.0 \%)$ & $148 / 1,350(11.0 \%)$ & N.S. \\
Anti-HDV+ & $104(7.6 \%)$ & $95 / 1,179(8,1 \%)$ & N.S. \\
Anti-HCV+ & $223(16.3 \%)$ & $83 / 1,277(6.5 \%)$ & $<0.01$ \\
Anti-HIV+ & $31(2.3 \%)$ & $18 / 889(2.0 \%)$ & N.S. \\
\hline
\end{tabular}

HDV: Hepatitis D virus; HCV: Hepatitis C virus; HIV Human Immunodeficiency virus.

$P<0.01)$ significantly decreased. No difference was observed in the proportion of $\mathrm{HBeAg}$ positive cases $(11.7 \%$ vs. $11.6 \%)$, anti-HDV positivity $(8.0 \%$ vs. $7.0 \%$ ) and HIV positivity (3.1\% vs. $2.6 \%$ ). (Data not shown).

Comparision of HBsAg positive female carriers 6 years apart evidences that the proportion of people born abroad (5.9\% vs. $10.0 \% ; P<0.01)$, and that of people HDV coinfected $(6.6 \%$ vs. $11.7 \% ; P<0.01)$ nearly doubled over time; while the proportion of incident cases $(20.8 \%$ vs. $12.4 \% ; P<0.01)$, and that of people HCV coinfected $(14.1 \%$ vs. $4.9 \% ; P<0.01)$ strongly decreased. The mean age of subjects (50.4 years vs. 49.0 years), the proportion of $\mathrm{HBeAg}$ positivity $(12.8 \%$ vs. $9.8 \%)$, that of HIV coinfected $(0.3 \%$ vs. $0.4 \%)$, and that of subjects with liver cirrhosis (14.6\% vs. $16.2 \%)$ did not show significant changes over time. (Data not shown).

\section{DISCUSSION}

Firstly, we would like to outline the decreasing proportion over time of incident cases in both genders, which is a further proof for the declining endemicity of HBV infection in Italy [Stroffolini 2005]. However, the higher proportion of incident cases in females than in males reflects the very high adherence to HBsAg screening for pregnant women during the third trimester of pregnancy. In fact, it has been recently shown that as many as $97.9 \%$ of both Italian and foreign pregnant women undergo to the screening [Spada et al., 2011].

The present study confirms previous observations that there is a propensity for chronic HBV infection as well as for more active liver disease in men [McMahon et al., 1990; Chen 1993; Poynard et al., 2001]. The greater progression of hepatic fibrosis and HCC in men may be due, at least in part, to a low production of estradiol, which is a potent endogenous antioxidant which suppresses hepatic fibrosis in animal models, and attenuates reduction of redox sensitive transcripion factors, hepatocyte apoptosis, and HSC activation by inhibiting a generation of reactive oxygen species in primary cultures [Shimizu et al., 2007]. Further study recently confirmed the protective role for estrogens against the development of HCC in female subjects [Wang et al., 2012]. Androgen, in contrast to estrogen, exerts an opposite effect on HBV transcription and replication. It has been shown that the androgen pathway can increase the transcription of $\mathrm{HBV}$ through direct binding of the androgen-responsive element sites in viral enhancer I, explaining a higher titer in male carriers and an increased risk of HCC [Wang et al., 2009]. Thus, sexual hormones have an opposite effect on liver carcinogenesis, in which androgen promotes

TABLE II. Comparison of Chronic HBsAg Carriers by Gender

\begin{tabular}{|c|c|c|c|}
\hline Factor & Males $(\mathrm{n}=2,000)$ & Females $(\mathrm{n}=762)$ & $P$ value \\
\hline Age (mean \pm SD) & $49.3 \pm 13.1$ & $49.7 \pm 14$ & N.S. \\
\hline Incident cases & $210 / 1,923(10.9 \%)$ & $123 / 737(16.6 \%)$ & $<0.001$ \\
\hline Born abroad & $101 / 1,991(5.1 \%)$ & $60 / 758(7.9 \%)$ & 0.03 \\
\hline $\mathrm{HBeAg}+$ & $229 / 1,966(11.6 \%)$ & $83(11.1 \%)$ & N.S. \\
\hline Anti-HDV+ & 139/1,851 (7.5\%) & 60/694 (8.6\%) & N.S. \\
\hline Anti-HCV+ & $235 / 1,911(12.3 \%)$ & $71 / 732(9.7 \%)$ & N.S. \\
\hline Anti-HIV+ & $47 / 1,629(2.9 \%)$ & $2 / 626(0.3 \%)$ & $<0.001$ \\
\hline HBV DNA ${ }^{\mathrm{a}, \mathrm{b}}$ undetectable & $286 / 876(32.6 \%)$ & $110 / 347(31.7 \%)$ & N.S. \\
\hline Diagnostic category $^{\mathrm{c}}$ & $277(13.9 \%)$ & $207(27.2 \%)$ & $<0.001$ \\
\hline Normal ALT & $1216(60.8 \%)$ & $426(55.9 \%)$ & \\
\hline Chronic hepatitis & $425(21.2 \%)$ & $117(15.3 \%)$ & \\
\hline Liver cirrhosis & $82(4.1 \%)$ & $12(1.6 \%)$ & \\
\hline $\mathrm{HCC}$ & & & \\
\hline
\end{tabular}

HCC: Hepatocellular Carcinoma; HDV: Hepatitis D virus; HCV: Hepatitis C virus; HIV Human Immunodeficiency virus.

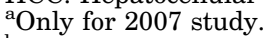

${ }^{\mathrm{b}}$ Only in untreated patients.

'The sex ratio $(\mathrm{M} / \mathrm{F})$ was 1.3 in normal ALT, 2.8 in chronic hepatitis, 3.6 in liver cirrhosis and 6.8 in HCC. 
TABLE III. Risk Factors Associated with Cirrhosis/HCC in HBsAg Positive Patients by Uni and Multivariate Analysis

\begin{tabular}{|c|c|c|c|c|c|}
\hline Variable & Chronic hepatitis & Cirrhosis/HCC & Crude OR (95\% CI) & Adjusted OR (95\% CI) & $P$-value \\
\hline \multicolumn{6}{|l|}{ Age (years) } \\
\hline$\leq 50$ & $1,247(58.7 \%)$ & $167(26.3 \%)$ & 1 & & \\
\hline$>50$ & $879(41.3 \%)$ & $469(73.7 \%)$ & $3.98(3.27-4.85)$ & $4.78(3.85-5.98)$ & $<0.001$ \\
\hline \multicolumn{6}{|l|}{ Gender } \\
\hline Female & $633(29.8 \%)$ & $129(20.3 \%)$ & 1 & & \\
\hline Male & $1,493(70.2 \%)$ & $507(79.7 \%)$ & $1.67(1.34-2.06)$ & $1.66(1.29-2.14)$ & $<0.001$ \\
\hline \multicolumn{6}{|l|}{ Anti-HCV } \\
\hline Negative & $1,821(90.0 \%)$ & $516(83.4 \%)$ & 1 & & \\
\hline Positive & $203(10.0 \%)$ & $103(16.6 \%)$ & $1,79(1.39-2.13)$ & $1.62(1.22-2.16)$ & 0.001 \\
\hline \multicolumn{6}{|l|}{ Anti-HDV } \\
\hline Negative & $1,822(93.9 \%)$ & $524(86.8 \%)$ & 1 & & \\
\hline Positive & $119(6.1 \%)$ & $80(13.2 \%)$ & $2.34(1.73-3.15)$ & $2.67(1.90-3.77)$ & $<0.001$ \\
\hline \multicolumn{6}{|c|}{ Alcohol (drinks/day) } \\
\hline 0 & $1,228(57.8 \%)$ & $292(45.9 \%)$ & 1 & 1 & \\
\hline$\leq 4$ & $750(35.2 \%)$ & $237(37.3 \%)$ & $1.33(1.09-1.61)$ & $0.95(0.76-1.12)$ & 0.7 \\
\hline$>4$ & $148(7.0 \%)$ & $207(16.8 \%)$ & $5.88(4.60-7.53)$ & $2.55(1.84-3.53)$ & $<0.001$ \\
\hline \multicolumn{6}{|l|}{ BMI $\left(\mathrm{kg} / \mathrm{m}^{2}\right)$} \\
\hline$<25$ & $1,100(53.4 \%)$ & $275(44.6 \%)$ & 1 & & \\
\hline $25-30$ & $781(37.9 \%)$ & $277(45.0 \%)$ & $1.43(1.18-1.73)$ & $1.16(0.94-1.44)$ & 0.16 \\
\hline$>30$ & $180(8.7 \%)$ & $64(10.4 \%)$ & $1.44(1.05-1.97)$ & $1.26(0.88-1.80)$ & 0.2 \\
\hline \multicolumn{6}{|c|}{ HBV-DNA (copies/mla ${ }^{a, b a, b}$} \\
\hline$<10^{3}$ & $429(46.8 \%)$ & $160(53.9 \%)$ & 1 & & \\
\hline $10^{3}-10^{4}$ & $152(16.6 \%)$ & $44(14.8 \%)$ & $0.78(0.53-1.14)$ & & \\
\hline$>10^{4}$ & $335(36.6 \%)$ & $93(31.3 \%)$ & $0.74(0.56-1.00)$ & & \\
\hline
\end{tabular}

Crude and adjusted Odds Ratios (OR) deriving from multiple logistic regression analysis. HCC, Hepatocellular carcinoma; HDV, Hepatitis $\mathrm{D}$ virus; HCV, Hepatitis $\mathrm{C}$ virus; BMI, Body mass index.

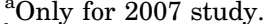

${ }^{\mathrm{b}}$ Only in untreated patients.

tumors, but estrogen protects against tumors, by regulating the HBV life cycle. HBV may be considered a sex hormone-responsive virus, which explains the sex disparity in the outcomes of chronic HBV infection.

According with the above-mentioned biological studies, our epidemiological survey shows that the male/female ratio was nearly fivefold higher in HCC cases than in subjects with normal ALT (6.8 vs. 1.3). This finding is in agreement with a previous Asian study showing a greater incidence of $\mathrm{HCC}$ in $\mathrm{HBsAg}$ positive men as compared to women, being the cumulative lifetime HCC incidence $27.4 \%$ in men and $7.9 \%$ in women, respectively [Huang et al., 2011]. Moreover, the present study, controlling by multiple logistic analysis for important risk factors associated with liver disease, shows that gender alone is truly responsible for the increasing ratios observed with increasing severity of liver disease confirming the relevance of molecular signaling studies.

Presence of coinfection with other viruses deserves some comment. No gender difference was observed regarding anti-HDV positivity. However, the proportion of males Delta coinfected was stable over time, but the corresponding figure for females increased over time, suggesting a potential survival phenomenon (i.e., a greater proportion of males than females more likely died for liver diseases HDV related). The very high proportion of $\mathrm{HBsAg}$ positive carriers tested for HDV reflects the great attention and awareness addressed to HDV infection in Italy.
No intergender difference was observed in the proportion of subjects HCV coinfected. However, in both genders, HCV infection significantly decreased over time, confirming the vanishing trend of the infection recently reported [Guadagnino et al., 2013]. Few subjects were anti-HIV coinfected, with a male predominance stable over time, reflecting more likely sources of exposure in male sex.

In conclusion, this survey evidences several differences in sex-related HBV chronic infection, with a remarkable higher proportion of males with more severe liver disease. We are aware that some risk factors associated with liver disease, such as HBV genotype or mutations, have not been controlled for because not available. It represents a potential limitation of the present study. However, the observed sex disparity in the outcome of chronic $\mathrm{HBsAg}$ carriers, even if derived from only an observational study, may support biological observation that HBV infection could be considered a sex-hormone responsive virus.

\section{REFERENCES}

Blumberg BS, Sutnick AI, London WT, Melartin L. 1972. Sex distribution of Australia antigen. Arch Intern Med 130:227-231.

Bruix J, Sherman M. 2005. Management of hepatocellular carcinoma. Hepatology 42:1208-1236.

Chang MH, Shau WY, Chen CJ, Wu TC, Kong MS, Liang DC, Hsu HM, Chen HL, Hsu HY, Chen DS. 2000. Taiwan Childhood Hepatoma Study Group. Hepatitis B vaccination and hepatocellular carcinoma rates in boys and girls. JAMA 284:30403042 . 
Chen DS. 1993. Natural history of chronic hepatitis B virus infection: New light on an old story. J Gastroenterol Hepatol 8:470-475.

Evans AA, Chen G, Ross EA, Shen FM, Lin WY, London WT. 2002. Eight-year follow-up of the 90,000-person Haimen City cohort: I. Hepatocellular carcinoma mortality, risk factors, and gender differences. Cancer Epidemiol Biomarkers Prev 11:369_ 376.

Gaiani S, Gramantieri L, Venturoli N, Piscaglia F, Siringo S, D'Errico A, Zironi G, Grigioni W, Bolondi L. 1997. What is the criterion for differentiating chronic hepatitis from compensated cirrhosis? A prospective study comparing ultrasonography and percutaneous liver biopsy. J Hepatol 27:979-985.

Guadagnino V, Stroffolini T, Caroleo B, Menniti Ippolito F, Rapicetta M, et al. 2013. Hepatitis $\mathrm{C}$ virus infection in an endemic area of Southern Italy 14 years later: Evidence for a vanishing infection. Dig Liver Dis 45:403-407.

Huang YT, Jen CL, Yang HI, Lee MH, Su J, Lu SN, Iloeje UH, Chen CJ. 2011. Lifetime risk and sex difference of hepatocellular carcinoma among patients with chronic hepatitis B and C. J Clin Oncol 29:3643-3650.

Lee CM, Lu SN, Changchien CS, Yeh CT, Hsu TT, et al. 1999. Age, gender, and local geographic variations of viral etiology of hepatocellular carcinoma in a hyperendemic area for hepatitis B virus infection. Cancer 86:1143-1150.

McMahon BJ, Alberts SR, Wainwright RB, Bulkow L, Lanier AP. 1990. Hepatitis B-related sequelae. Prospective study in 1400 hepatitis B surface antigen-positive Alaska native carriers. Arch Intern Med 150:1051-1054.

Poynard T, Ratziu V, Charlotte F, Goodman Z, McHutchinson J, Albrecht J. 2001. Rates and risk factors of liver fibrosis progression in patients with chronic hepatitis C. J Hepatol 34: $730-739$.
Sagnelli E, Stroffolini T, Mele A, Imparato M, Almasio PL. 2008. Italian Hospitals' Collaborating Group. Chronic hepatitis B in Italy: New features o fan old disease - approaching the universal prevalence of hepatitis B e antigen - negative cases and the eradication of hepatitis D infection. Clin Infect Dis 46:110113.

Shimizu I, Kohno N, Tamaki K, Shono M, Huang HW, et al. 2007. Female hepatology: Favorable role of estrogen in chronic liver disease with hepatitis B virus infection. World J Gastroenterol 13:4295-4305.

Spada E, Tosti ME, Zuccaro O, Stroffolini T, Mele A 2011. Collaborating Study Group. Evaluation of the compliance with the protocol for preventing perinatal hepatitis B infection in Italy. J Infect 62:165-171.

Stroffolini T. 2005. The changing pattern of hepatitis B virus infection over the past three decades in Italy. Dig Liver Dis 37:622-627.

Stroffolini T, Almasio PL, Sagnelli E, Mele A, Gaeta GB, Italian Hospitals' Collaborating Group et al. 2009. Evolving clinical landscape of chronic hepatitis B: A multi center Italian study. J Med Virol 81:1999-2006.

Taylor BC, Yuan JM, Shamliyan TA, Shaukat A, Kane RL, Wilt TJ. 2009. Clinical outcomes in adults with chronic hepatitis B in association with patient and viral characteristics: A systematic review of evidence. Hepatology 49:985-995.

Wang SH, Yeh SH, Lin WH, Wang HY, Chen DS, Chen PJ. 2009. Identification of androgen response elements in the enhancer I of hepatitis $B$ virus: A mechanism for sex disparity in chronic hepatitis B. Hepatology 50:1392-1402.

Wang SH, Yeh SH, Lin WH, Yeh KH, Yuan Q, et al. 2012. Estrogen receptor repress transcription of $\mathrm{HBV}$ genes via interaction with hepatocyte nuclear factor 4. Gastroenterology 142:989998. 